\title{
Genetic diversity of Triticum araraticum assessed using cytogenetics
}

\author{
Badaeva E.D. ${ }^{1 *}$, Özkan H. ${ }^{2}$, Kilian B..$^{3,4}$ \\ ${ }^{1}$ N.I. Vavilov Institute of General Genetics, Russian Academy of Sciences, Moscow, Russia \\ ${ }^{2}$ Department of Field Crops, Faculty of Agriculture, University of Çukurova, Adana, Turkey \\ ${ }^{3}$ Leibniz Institute of Plant Genetics and Crop Plant Research (IPK), Gatersleben, Germany \\ ${ }^{4}$ Global Crop Diversity Trust, Bonn, Germany \\ * email: katerinabadaeva@gmail.com
}

Triticum araraticum Jakubz. is wild tetraploid wheat with the genome constitution GGA $^{t} \mathrm{~A}^{\mathrm{t}}$. Distribution area of $T$. araraticum covers the northern part of the Fertile Crescent: northern Iraq, eastern and southeastern Turkey, northwestern Iran, Armenia, Azerbaijan and northern Syria. Its domesticated derivative T. timopheevii was found only in Western Georgia. Genetic variation of T. araraticum and its relationships with T. timopheevii were accessed using cytogenetic analyses. Altogether 370 genebank accessions of $T$. araraticum (representing 391 genetically distinct biotypes) and 17 T. timopheevii were scored using C-banding and 92 accessions were selected for FISH with polymorphic DNA probes pSc119.2, Spelt-1, Spelt-52. All markers revealed high genetic diversity of $T$. araraticum and broad translocation polymorphisms. Normal karyotypes were found in $44.6 \%$ of $T$. araraticum accessions, whereas $55.4 \%$ accessions carried chromosomal rearrangements, represented by 76 variants. These were single (44 variants) and multiple translocations (24), paracentric (1) and pericentric (7) inversions. Translocation spectra were specific for different geographical regions. Irrespective of karyotype structure, C-banding analysis identified three well-separated clusters, which were designated ARA-0, ARA-1, and TIM, each corresponding to plastogroups G-2, G-1+G-3 and TIM, according to Mori et al. (2009). The ARA-0 group grows over the entire distribution range and exhibits the broadest genetic diversity. The ARA-1 group is found in southeast Turkey and northern Syria, where the areas of T. araraticum and T. dicoccoides overlap. Triticum timopheevii seemed to be closer related to ARA-1, than to ARA-0 group.

Acknowledgments: This work was supported by grant from the Russian State Foundation for Basic Research (project 20-04-00284). 